\title{
THE CHINESE ARMED FORCES \\ IN THE 21st CENTURY
}

\author{
Edited by \\ Larry M. Wortzel
}

December 1999 